\title{
FUSION-BASED INTELLIGENT SUPPORT \\ FOR LOGISTICS MANAGEMENT
}

\author{
Alexander Smirnov, Mikhail Pashkin, Nikolai Chilov, \\ Tatiana Levashova, and Andrew Krizhanovsky \\ St.Petersburg Institute for Informatics and Automation of the Russian Academy \\ of Sciences, 39, 14-th Line, 199178, St.-Petersburg, RUSSIA \\ \{smir, michael,nick,oleg,aka\}@mail.iias.spb.su
}

\begin{abstract}
Since the Internet has grown an easily accessible and popular place for business applications the problem of knowledge integration based on using Web tools and dealing with knowledge representation and processing has become actual. The paper presents a knowledge fusion agent as a part of multiagent system addressing knowledge logistics. This agent is based on a constraint satisfaction technology. The task statement for knowledge fusion agent is presented in an ontology form. This flexible task representation via transparent and open ontology formalism enables creating routing plans for a transportation system of a virtual production network.
\end{abstract}

\section{INTRODUCTION}

Rapid development of the Internet has caused a huge amount of information about different problem areas to become available for users. Since the information is represented in various formats and by different tools, the problems of format compatibility, search tools implementation, recognition and fusion of knowledge from distributed sources/resources have become critical. The necessity of the knowledge fusion $(\mathrm{KF})$ approach development for global understanding of going on processes and phenomena, dynamic planning and global knowledge exchange $h$ as developed. The KF methodology is a new direction of knowledge management in the part of knowledge logistics (Smirnov et al., 2003a).

In nowadays conditions knowledge is becoming an important resource. The main characteristics of it are the following: (i) knowledge is a critical source of long-term competitive advantage; (ii) knowledge is more powerful than natural resources; (iii) knowledge resource has cost, location, access time and life-time; (iv) knowledge worker is an owner of knowledge and a member of a team/group.

Related to this, along with development of computing machinery and information technologies, there arose a need of systems working with knowledge, i.e. dealing with knowledge creation, classification, synthesis, analysis, storage, search and mapping. 
Intelligent agents are a very hot research topic that significantly changed the way distributed systems are working. Multi-agent system technology was chosen as the basis for KF systems (Smirnov et al., 2002).

Logistics systems play an important role in manufacturing companies, especially based on the concept of the virtual production network (Golm \& Smirnov, 2000). An intelligent support, based on technologies of Web intelligence, intelligent agents, and open services, may significantly enhance the logistics system abilities (e.g., reduce costs and times of delivery). Therefore the paper proposes an application of knowledge logistics as an intelligent service for creation efficient routing plans (as one of the major logistics tasks in virtual production network management) under given constraints and preferences. This application is illustrated via a case study of delivering goods to customers.

Numerous logistics techniques are known, from the traveling salesman problem to complex dynamic problems. Vehicle rooting problem (VPR) as part of logistics has received a lot of attention in the literature because many real world transportation problems are related to it (Tarantilis et al., 2004; Ruiz et al., 2004). VPR can be briefly described as a set of $N$ clients or customers with known demands $d_{i}, i \in 1, \ldots, N$, that have to be served from a central depot with a fleet of $t$ delivery trucks of known capacity $Q$. VPR is a problem of a high computational complexity (NP-hard), so the large number of approaches focusing on using heuristics and constrained satisfaction techniques. An integration of ontology management and constraint satisfaction solving VPR is presented in the paper.

The paper is organized as follows. Section 2 elucidates the knowledge logistics concerned with the ontology approach. Section 3 presents the KF agent design, implementation and its decisive role in the integration of ontology management and constraint satisfaction. Section 4 describes a case study. Main features of the system "KSNet" due to using the KF agent are presented in the conclusion.

\section{ONTOLOGY-DRIVEN KNOWLEDGE LOGISTICS}

Knowledge logistics addresses the problem of acquisition of the right knowledge from distributed sources, its integration and transfer to the right person within the right context, at the right time, for the right purpose. This problem in the approach is considered a s a c onfiguration of n etwork that i ncludes end-users, loosely c oupled knowledge sources, and a set of tools and methods for knowledge processing located in e-business environment. Such network of loosely coupled sources was referred to as knowledge source network or "KSNet".

The application of intelligent agents representing their knowledge via ontologies (Weiss, 2000) was motivated by the need of knowledge logistics systems for flexibility, scalability, and customizability. The multiagent system architecture based on the FIPA Reference Model (FIPA, 2004) was chosen as a technological basis for the definition of agents' properties and functions since it provides standards for heterogeneous interacting agents and agent-based systems, and specifies ontologies and negotiation protocols.

The formalism of object-oriented constraint networks (Smirnov et al., 2003a) was chosen as the abstract model for ontology representation. According to the formalism knowledge can be described by classes, attributes, domains, constraints, 
and methods. This perspective of knowledge representation correlates well with the semantic metadata representation concept being developed under the Semantic Web project (Semantic Web, 2004).

The thorough comparison of multi-agent systems (KRAFT, InfoSleuth) designed for knowledge fusion operations vs. the system "KSNet" has been done in previous works (Smirnov et al., 2001). The system "KSNet" has a distributed multiagent architecture (Smirnov et al., 2002). Components of the system can be allocated at different hosts and connected via TCP/IP protocol. Users and experts can work with the system via a Web-based interface.

\section{KNOWLEDGE FUSION AGENT}

The KF agent is responsible for KF operations. It is shown below that KF agent has a distributed nature and uses a constraint satisfaction technology.

\subsection{Solver Middleware Design}

The system uses ontologies for user request processing. A user request in free form defines both the problem statement and what data has to be retrieved from ontology library (OL) and from knowledge sources (KSs). Thereby the problem statement is changed from one r equest to a nother. The "on-the-fly" c ompilation mechanism in combination with ILOG (ILOG, 2004) is proposed to solve these varying problems. In a rough outline this "on-the-fly" compilation mechanism is based on the following concepts (Figure 1):

- a pre-processed u ser r equest defines (1) which o ntologies are to be extracted from an ontology library (OL), and (2) which KSs are to be used;

- $\mathrm{C}++$ code is generated on the basis of information extracted from (1) the user request (goal, goal objects, etc.), (2) appropriate ontologies (classes, attributes, and constraints), and (3) suitable KSs;

- the compilation is performed in an environment of the prepared in advance $\mathrm{C}++$ project;

- failed compilations/executions do not fail the system work in whole; an appropriate message for the user is generated.

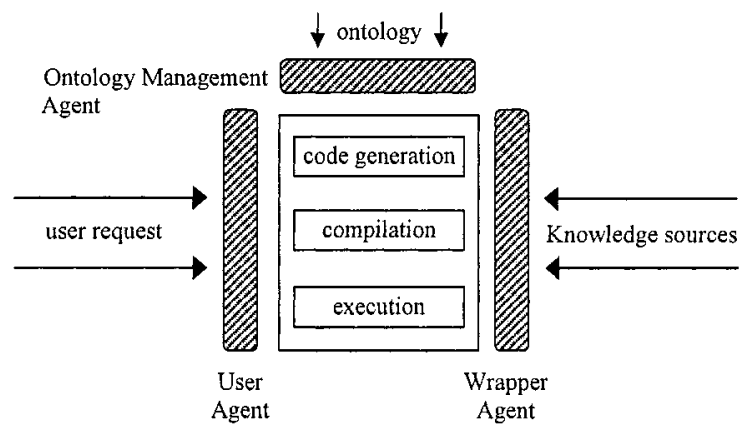

Figure 1 - Solver "on-the-fly" compilation mechanism 


\subsection{Solver Middleware Implementation}

The KF agent is directly coupled with the solver. The described earlier mechanism of the "on-the-fly" compilation assumes that the $\mathrm{KF}$ agent gathers required data from other the system "KSNet" agents (translation agent, ontology management agent, configuration agent, and wrappers), generates a solver (performs the "on-the-fly" compilation), and launches the solver to generate a solution. In more detailed fashion this particular task performed by the KF agent requires: (i) a pre-processed user request prepared by the translation agent; (ii) an appropriate ontology demanded from the OL found and extracted by the ontology management agent; (iii) knowledge received from KSs by wrappers.

ILOG Solver has been chosen as a constraint solver because it has the following valuable features: (i) it is based around some $\mathrm{C}++$ libraries; (ii) it has functionalities for managing constraints; (iii) it has solver; (iv) user defines solving process; (v) user defines constraints propagation mechanisms.

The KF agent and ILOG Solver interact via the XML-RPC (XML-RPC, 2004) protocol (remote procedure calling via HTTP as a transport and XML as an encoding). XML-RPC is designed to be as simple as possible, while allowing complex data structures to be transmitted, processed and returned.

$\mathrm{XmlRpc}++$ library $(\mathrm{XmlRpc}++, 2004)$ is used as an implementation of the $\mathrm{XmlRpc}$ protocol written in $\mathrm{C}++$. $\mathrm{XmlRpc}++$ is designed to make it easy to incorporate $\mathrm{XmlRpc}$ client and server support into $\mathrm{C}++$ applications.

The agent performs KF operations based on Application Ontology (AO) and knowledge acquired from KSs. The implementation of the KF agent uses such fundamental ideas of programming languages as object-oriented approach and logic programming. ILOG Configurator (ILOG, 2004) was chosen as a generic tool for object-oriented constraint programming. It provides a library of re-usable and maintainable $\mathrm{C}++$ classes. These classes define objects in the application domain in a natural and intuitively way so that it is possible clearly distinguish the problem representation from the problem resolution. Therefore, if a problem statement changes then it is not necessary to rewrite the entire code as in case of "pure" $\mathrm{C}++$. In the given case the problem statement is defined by data retrieved from OL, therefore the problem of minimal code modification, fast and error-free is the important task here.

The essence of the proposed "on-the-fly" compilation mechanism is to write AO elements (classes, attributes, constraints) to a $\mathrm{C}++$ file directly. The $\mathrm{KF}$ agent creates a $\mathrm{C}++$ file based on these data and inserts program source code into a program (Microsoft Visual Studio project) prepared in advance. The program is compiled in order to create an executable file in the form of dynamic-link library (DLL). After that the KF agent calls a function from DLL to solve the task. The UML sequence diagram (Figure 2) shows the KF agent's scenario at the stages of knowledge obtaining, solver compilation and execution.

The KF agent uses the mentioned technique of the dynamic code generation (with ILOG Configurator commands embedded) to produce a solution set satisfying requirements of the $\mathrm{u}$ ser request and $\mathrm{AO}$ elements (classes, attributes, constraints, etc.).

The generated code $(\mathrm{C}++$ file $)$ consists of several parts: 
- the ontology management agent passes a part of the program based on data from the OL;

- the wrapper passes a part of the program using local/remote KSs;

- the KF agent generates a part of the program based on user request processing as well as user requirements;

- a predefined part of code (unchangeable): strategy definition and automatic answer generation.

Thus the $\mathrm{C}++$ file is created on the basis of a special template. This template allows researchers and developers to comprehend and realize in more explicit and welldefined form: (i) what information is needed to solve a task; (ii) which KSs are required; (iii) which agent is responsible for delivering particular specified information block.

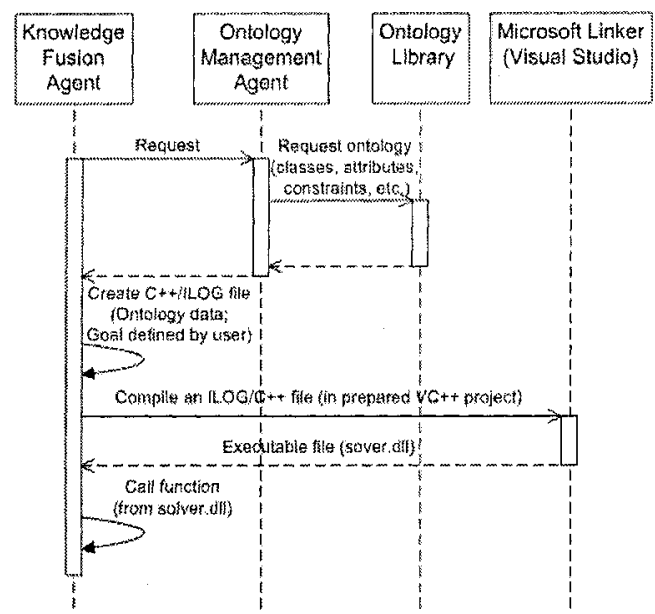

Figure 2 - The UML sequence diagram shows the KF agent scenario

\section{CASE STUDY}

A simplified version of the ontology describing a virtual production network used for the example is demonstrated in Figure 3. Based on this ontology the task ontology of the "configuration" task is built. The tasks "Staff definition" and "Cargo Allocation" are shown as dashed since they were out of the scope of the project. The other tasks were solved using ILOG (Solver, Configurator and Dispatcher) and specially written procedures (e.g., procedure that estimates impact of the weather on roads availability and transportation time).

The task of allocation is devoted to finding the most appropriate location for a distribution center considering such factors as location of the producers, nearby cities and towns, availability of facilities (e.g., communications), and decision maker's choice and priorities. This task was solved by ILOG Solver. The task of capacity and bill-of-material (BOM) definition was based on predefined information and specially created procedures taking into account such factors as demand forecast and others. The objective of the task of resource allocation is to define the most 
appropriate suppliers taking into account their capacities, prices, transportation costs, availability, etc. This task w as solved using ILOG Configurator. The above tasks were described in detail in (Smirnov et al., 2003b; Smirnov et al., 2003c).

The task of routing, the paper concentrates on, is devoted to finding a paretooptimal set of routes of delivery of products from selected suppliers considering such factors as communications facilities (e.g., locations of airports, roads, etc.), their conditions (e.g., good, damaged or destroyed roads), weather conditions (e.g., rains, storms, etc.) and decision maker's choice and priorities.

Presented example illustrates finding a routing plan for the same conditions but with different user preferences, namely: minimize time; minimize time, then costs; minimize both time and costs; minimize costs, then time; minimize costs. In (Figure 4) results for the case minimize costs are presented. For illustration of the results a map is generated that uses the following notations. Green dots are the cities of the region. The city with red edge (Aida) is the city where the dealer is located. The cities with blue edges are the cities where customers are located (Libar, Higgville, Ugwulu, Langford, Nedalla, Laki, Dado). Transportations routes are shown as lines. The grey lines are routes that are not used for transportation in the solution, the blue lines routes used for transportation, and the red lines are routes unavailable due to weather or for some other reasons. E.g., the routes through the city of Zaribe are not available because of the flooding. The colored trucks denote the routes of particular vehicles/vehicle groups.

Figure 5 represents a comparison of the routing plans created for different criteria. As it can be seen while importance of one of the parameters increases (e.g., importance for costs increases from left to right) the value of the parameter decreases (the red line with diamonds for the costs) and vice versa (the green line with squares for the time).

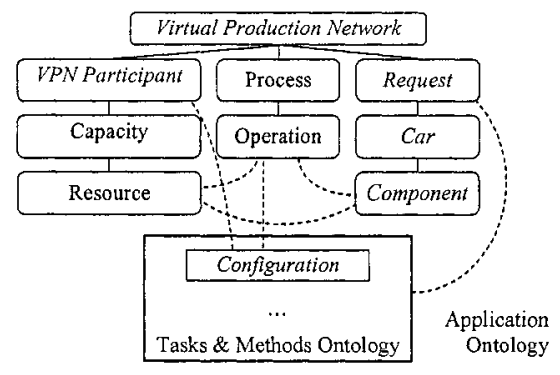

Figure 3 - Virtual Production Network ontology

\section{CONCLUSION}

The system "KSNet" where the presented KF agent plays one of the key roles has the following abilities: (i) ontology representation paradigm facilitates to process and understand natural language; (ii) ontology library based on the common vocabulary and notation can be considered as a dynamically created source of metaknowledge, (iii) user profiles and request ontologies support the personalization requirement; (iv) translation of ontologies from advanced formats) 


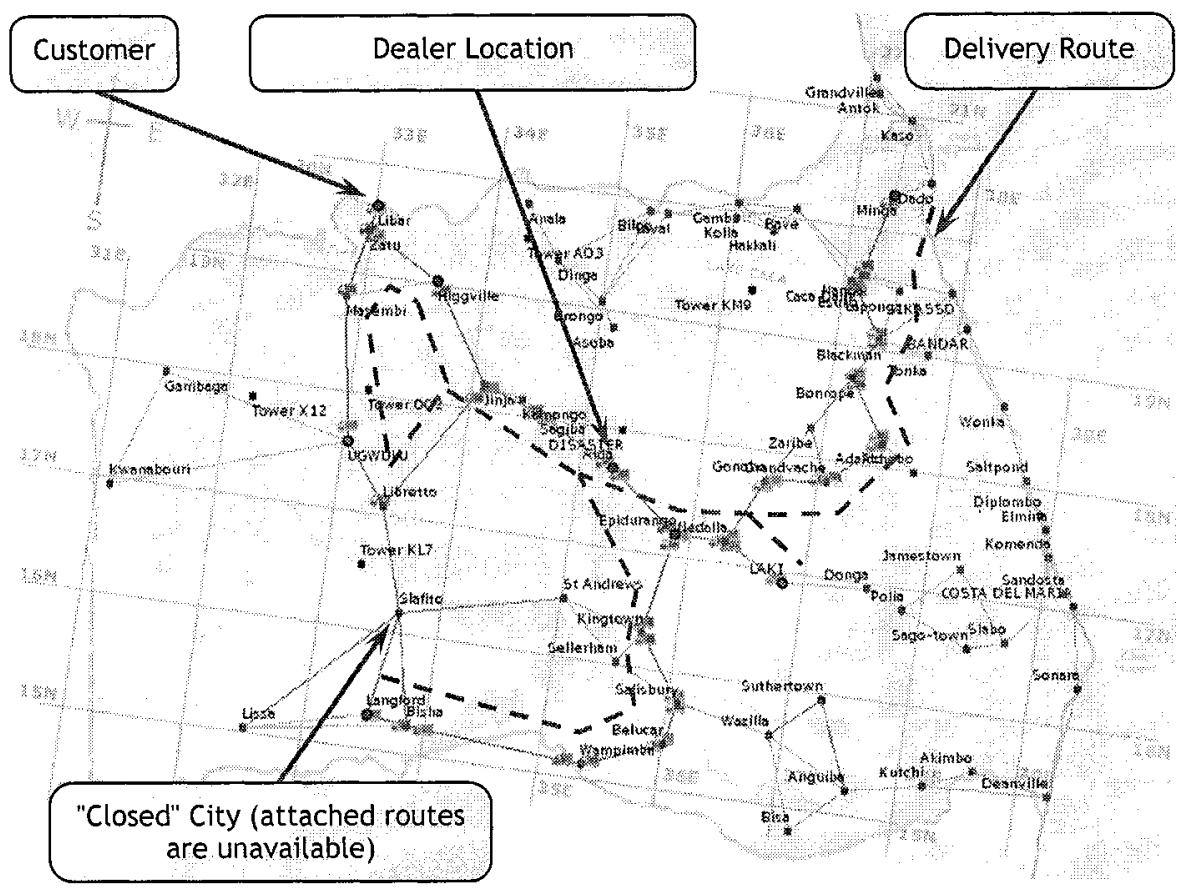

Figure 4 - Routing plan for the minimize costs preference (in this solution one vehicle/vehicle group is used to provide minimum of costs)

\section{Route Plans for Different Criteria}

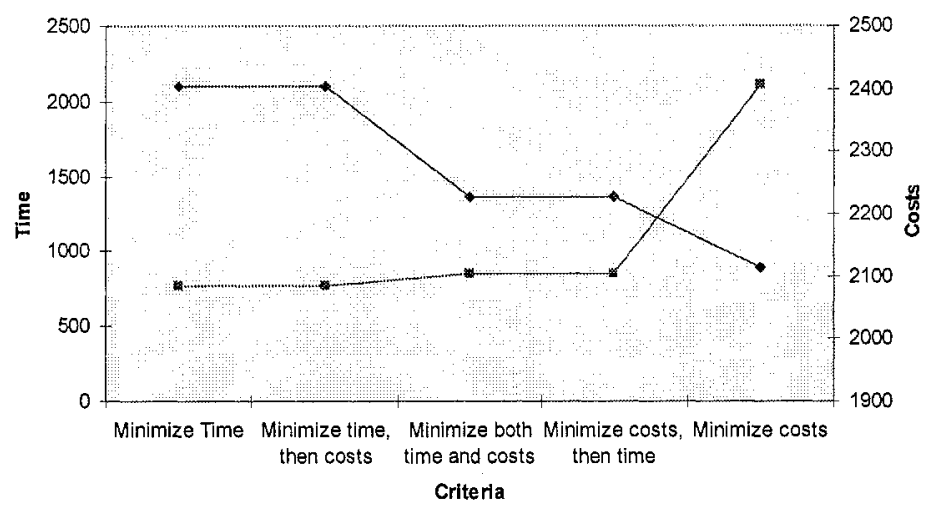

$\ldots-$ Time ...... Costs

Figure 5 - Routing plans for different criteria (time and costs minimization preferences) 
(e.g., DAML+OIL) into internal representation and out of it enables knowledge sharing and reuse; (v) the technology of constraint satisfaction \& propagation enables to perform automatic reasoning on the Web.

Using the knowledge fusion agent based on the mechanism of the "on-the-fly" compilation allows generating new knowledge that is not available in existing knowledge sources independently on the application domain of the current problem. Here presented approach can be used for solving such real-world tasks as logisitics, configuration, management in various application domains of distributed nature including manufacturing, transportation, healthcare, etc.

\section{REFERENCES}

1. Bowman M., Tecuci G., and Ceruti M., Application of Disciple to Decision Making in Complex and Constrained Environments, Proceedings of the 2001 IEEE Systems, Man, and Cybernetics Conference 2001

2. FIPA Foundation for Intelligent Physical Agents Documentation 2004. URL: http://www.fipa.org

3. Golm F., and Smirnov A. Virtual Production Network Configuration: ACS-approach and tools // Advances in Networked Enterprises: The Proceedings of the 4th IEEE/IFIP International Conference on Information Technology for Balanced Automation Systems in Production and Transportation (BASYS 2000). Berlin: K luwer Academic Publishers; B osten/Dordrecht/London, $2000 ; 103-110$.

4. ILOG Corporate Web-site, 2004. URL: hitt://www.ilog.com

5. Ruiz R., Maroto C. and Alcaraz J. A decision support system for a real vehicle routing problem, European Journal of Operational Research 2004; 153(3):593-606.

6. The Semantic Web Community Portal 2004; URL: http://www.semanticweb.org

7. Smirnov A., Pashkin M., Chilov N., \& Levashova, T. Multi-Agent Architecture for Knowledge Fusion from Distributed Sources. Proceedings of 2nd International Workshop of Central and Eastern Europe on Multi-Agent Systems (CEEMAS'2001). Krakow, Poland, September 26-29, $2001 ; 403-412$

8. Smimov A., Pashkin M., Chilov N. \& Levashova T. Agent-Based Knowledge Fusion in Scalable Information Environment: Major Principles and System Framework. Proceedings of the First International ICSC Congress on Autonomous Intelligent Systems (ICAIS'2002), ISBN: 3-90645430-4, Geelong, Australia, 2002.

9. Smirnov A., Pashkin M., Chilov N., Levashova T. and Haritatos F. Knowledge Source Network Configuration Approach to Knowledge Logistics. International Journal of General Systems. Taylor \& Francis Group 2003; 32(3): 251-269.

10. Smirnov, A. V., Pashkin, M. P., Chilov, N. G., Levashova, T, V. Agent-Based Support of Mass Customization for Corporate Knowledge Management. In: Engineering Applications on Artificial Intelligence $2003 ; 16(4): 349-364$.

11. Smimov, A. V., Pashkin, M. P., Chilov, N. G., Levashova, T. V. Knowledge Logistics in Information Grid Environment. The special issue "Semantic Grid and Knowledge Grid: The NextGeneration Web" (H. Zhuge, ed.) of International Journal on Future Generation Computer Systems 2003, $20(1): 61-79$.

12. Tarantilis C.D., Diakoulaki D. and Kiranoudis C.T. Combination of geographical information system and e fficient routing a lgorithms for real $l$ ife $d$ istribution o perations, European J ournal of Operational Research 2004; 152(2) 437-453.

13. XML-RPC Web-site, 2004. URL: http://www.xmlrpc.org

14. XmlRpc++ Library Web-site, 2004. URL: http://xmlrpcpp.sourceforge.net

15. Weiss, G. (ed.): Multiagent Systems: a Modern Approach to Distributed Artificial Intelligence. The MIT Press, Cambridge, Massachusetts, London, 2000. 